\title{
Competition and cuckoldry: estimating fitness of alternative reproductive tactics in plainfin midshipman
}

\author{
Karen M. Cogliati ${ }^{\mathrm{a}, *}$, Sigal Balshine ${ }^{\mathrm{a}}$ and Bryan D. Neff ${ }^{\mathrm{b}}$ \\ ${ }^{a}$ Department of Psychology, Neuroscience and Behaviour, McMaster University, \\ Hamilton, ON, Canada L8S 4K1 \\ ${ }^{\mathrm{b}}$ Department of Biology, Western University, London, ON, Canada N6A 5B7 \\ *Corresponding author's e-mail address: cogliakm@mcmaster.ca
}

Accepted 15 January 2014; published online 17 February 2014

\begin{abstract}
There has been much debate about how male alternative reproductive tactics (ARTs) evolve. In particular, researchers question whether ARTs have evolved as a conditional, 'best of a bad job' strategy where one tactic has higher fitness than the other, or whether they have evolved as a result of a genetic polymorphism where both tactics have equal fitness. Despite the large number of species known to have ARTs, tests of equal fitness between tactics have only been conducted in a handful of species. We tested the prediction of equal fitness using the plainfin midshipman (Porichthys notatus), a species with two well characterized male ARTs: guarding type I males and cuckolding type II males. We collected data across three years and three sampling locations to determine the proportion of each reproductive tactic, as well as the proportion of offspring sired by each male type using microsatellite markers. Our analysis suggests that males adopting the conventional guarding tactic likely have higher fitness compared to males adopting the cuckolder type II tactic. Also, we show that the guarding male tactic is able to gain paternity through cuckoldry, and that these males, who sometimes guard and sometimes cuckold, are responsible for the majority of paternity lost within nests. Indeed, the classic cuckolding type II males were responsible for only a small fraction of the paternity lost. These results highlight the degree of flexibility in male behaviour even among individuals adopting the same male tactic. Taken together, our results provide the first exploration of the evolution of male ARTs in plainfin midshipman and, given the tractability of midshipman system, a valuable next step will be to look for gene-by-environment interactions on tactic development and expression.
\end{abstract}

\section{Keywords}

sneaker males, guarder males, conditional strategy, life history model, genetic polymorphism, Porichthys notatus. 


\section{Introduction}

Alternative reproductive tactics (ARTs) occur when there are two or more reproductive phenotypes within a population, each attempting to maximize reproductive success using different behaviours (Taborksy et al., 2008). Most commonly reported in males, one morph, sometimes called guarding, parental, territorial, bourgeois, or type I, is typically associated with delayed sexual maturation, showy displays used to court females and deter rivals, and in some cases paternal care. The alternative male morph, called cuckolder, sneaker, parasitic, or type II, is more often associated with precocial maturation, and does not invest in courtship or paternal care, instead gaining reproductive success in sneaky or coercive ways. Intense male-male competition and large variation in male reproductive success are thought to select for the evolution of ARTs (Gross, 1996; Tabosky et al., 2008). These conditions promote the evolution of ARTs by providing some males a means to gain reproductive success by pursuing an alternative tactic.

Two mechanisms have been put forth to explain the evolution of alternative reproductive tactics. The first hypothesis argues that a genetic polymorphism is responsible for the expression of two or more tactics, and that different alleles at a single gene determine which tactic is expressed. Game theory shows that negative frequency dependent selection maintains the evolutionary stability of the alternative tactics, with each tactic having equal fitness (Maynard Smith, 1982; reviewed in Gross, 1996). The frequency dependence ensures stability because when one of the alternative tactics is rarer, it has higher fitness leading to an increase in frequency of the underlying allele in the population. A genetic polymorphism has been implicated as the mechanism responsible for ARTs in several species that include the ruff (Philomachus pugnax; Lank et al., 1995), a marine isopod (Paracerceis sculpta; Shuster, 1989; Shuster \& Wade, 1991), and a swordtail (Xiphophorus nigrensis; Ryan et al., 1992). The second hypothesis proposes that male tactics depend on individual differences in condition or status and hence on environmental and social influences (reviewed in Gross, 1996; Oliveira et al., 2008). In a conditional strategy, tactics are expressed based on a single gene or genes that code for a 'decision rule', where a male adopts the reproductive tactic that maximizes his fitness based on his condition or status at some critical point during development (Gross, 1996; Piche et al., 2008; Tomkins \& Moczek, 2009; Roff, 2011). An individual's status can be influenced by environmental effects prior to tactic divergence, including population density, the 
size of the individual, and other biotic and abiotic conditions (Emlen, 2008). Thus, individuals may develop into different tactics if they experience divergent environmental or growing conditions. Because each male utilizes the best tactic for his own situation, in a conditional strategy, the reproductive tactics can have unequal fitness and negative frequency-dependent selection is not required (Gross, 1996). A conditional strategy has been implicated as the mechanism responsible for ARTs in species that include the scarab dung beetle (genus Onthophagus; Eberhard, 1982; Emlen, 1994) and scorpionfly (Panorpa sp.; Thornhill, 1981).

Gross \& Charnov (1980) developed a life history model to calculate the relative fitness of each tactic and thus to differentiate between ARTs caused by genetic polymorphism or a conditional strategy. In their model, the proportion of males that develop into each male type in the population as well as the proportion of all offspring that are fertilized by each male type are calculated. If the proportion of males adopting each tactic is equal to the proportion of offspring sired by males using that tactic, then the tactics have equal fitness and a genetic polymorphism is inferred. If the tactics have unequal fitness, then a conditional strategy is inferred. Based on their initial application of the model, Gross \& Charnov (1980) determined that parental and cuckolder males in bluegill sunfish (Lepomis macrochirus) had equal fitness. However, with improved paternity data and controlled breeding experiments, Neff \& Lister (2007) calculated that cuckolder males had higher fitness relative to parental males, suggesting instead a conditional strategy. Because of the challenges associated with measuring reproductive success and the frequency of each tactic in wild populations, models to test equal fitness have only been applied to a small number of species (Gross \& Charnov, 1980; Shuster \& Wade, 1991; Ryan et al., 1992; Sinervo \& Lively, 1996; Neff \& Lister, 2007; Rios-Cardenas \& Webster, 2008).

Armed with paternity data and multi-year population sampling, we applied the Gross \& Charnov (1980) life history model to another species with well-characterized alternative reproductive tactics, the plainfin midshipman (Porichthys notatus). This species is a nocturnally active deep-water marine fish distributed along the Pacific coast of North America (Hubbs, 1920; Arora, 1948; Miller \& Lea, 1972). There are two distinct male reproductive tactics in this species known as guarding 'type I' and sneaker 'type II' males (Brantley \& Bass, 1994). At the onset of the breeding season in early spring, all reproductive adults undergo a large-scale vertical migration from deep 
waters $(>200 \mathrm{~m})$ to the spawning grounds in calm, rocky shores in the intertidal zone (Arora, 1948; Miller \& Lea, 1972). Type I males aggressively compete for limited nest sites, and the largest males typically win the largest nest sites (DeMartini, 1988). Guarding type I males use their sonic muscles, which are attached to their swim bladder, to acoustically court females (Ibara et al., 1983; Bass, 1992; Brantley et al., 1993). Females are attracted to these courtship songs and will produce a single clutch of up to 300 eggs (each 5-7 mm in diameter) per year, which are released into a single male's nest (DeMartini, 1990; authors unpublished data). Females and sneaker type II males are often sampled in the nests of guarding type I males, because spawning takes several hours to complete (Brantley \& Bass, 1994). Type I males will remain in the nest and continue to court and spawn with additional females until their nest is filled with multiple 'age cohorts' that are typically at different developmental phases (Arora, 1948; DeMartini, 1988; Brantley \& Bass, 1994; Cogliati et al., 2013). In contrast, sneaker type II males do not guard nests or court females, and have limited sonic muscle development. Instead, they steal fertilizations from guarding type I males by either sneaking into the nest when a female is present, and also by fanning their sperm into the nest from the periphery. Interestingly, type I males are known to be behaviourally flexible, and can also facultatively adopt cuckolding behaviour, particularly when nesting sites are severely limited or when they have no young in their own nests (Lee \& Bass, 2004; K.C., personal observation). Finally, nest takeovers have also been observed in plainfin midshipman, where guarding type I males may usurp another type I male from his nest and continue to guard the offspring of the previous nest owner (Brantley \& Bass, 1994; Cogliati et al., 2013).

There are several features that distinguish reproductively mature type I from type II males, including a number of behavioural, morphological, and neurobiological traits (Bass, 1990, 1992, 1993; Brantley \& Bass, 1994). Bass et al. (1996) have argued, based on differences in neuronal structures and on otolith growth patterns, that type I and type II males are mutually exclusive endpoints and not simply two parts of a single ontogenetic sequence. Thus, type I and type II male tactics represent two distinct life histories. Tactic divergence occurs at an early age of development (approximately 200 days), with type I males reaching sexual maturity three to four months after type II males (Bass et al., 1996; Bass, 1996). Based on the early differentiation in brain and muscle structures between the two tactics, Bass (1996) had 
initially argued that the tactics must be genetically determined. However, further research by this research group showed that male density during development affected morph outcomes (Foran, 1998). When male plainfin midshipman were reared in tanks under high density, a greater proportion of males developed into type II males as compared to tanks in which fish were reared in low density conditions, suggesting that the two morphs come about as a result of a conditional strategy (Foran, 1998). Thus, it remains unclear if the two reproductive tactics in midshipman represent alternative strategies based on a genetic polymorphism with equal fitness or a single conditional strategy based on a genetic monomorphism with unequal fitness.

In this study, we examined the fitness of the two male reproductive tactics in plainfin midshipman. We measured the frequency of both male tactics from a population in British Columbia over a number of years, and combined these estimates with paternity data for nest-guarding males from this same population, previously published in Cogliati et al. (2013). Additionally, here, for a subset of the nests, we also conducted extensive population-level sampling so that we could estimate the extent of cuckoldry by type I males. Finally, we used these data to apply the Gross \& Charnov (1980) life history model to determine if alternative reproductive tactics in plainfin midshipman have evolved as a genetic polymorphism or as a conditional strategy.

\section{Methods}

\subsection{Field collections}

In 2008, 2009 and 2010, between May and July, we monitored 473 plainfin midshipman nests during low tide in the intertidal zone on three rocky beaches in British Columbia (Ladysmith Inlet: $49^{\circ} 01^{\prime} \mathrm{N}, 123^{\circ} 83^{\prime} \mathrm{W}$; Mill Bay: $48^{\circ} 63^{\prime} \mathrm{N}, 123^{\circ} 53^{\prime} \mathrm{W}$; and Crescent Beach: $\left.49^{\circ} 04^{\prime} \mathrm{N}, 122^{\circ} 88^{\prime} \mathrm{W}\right)$. These sites represent a single breeding population with no genetic differentiation (Suk et al., 2009; Cogliati et al., data not shown). This population has been extensively monitored since 2007 and shows stable numbers of reproductively mature adults and a stable ratio of male types (Table 1). At each nest, we sexed all fish based on the shape of the urogenital papilla (blunt in females, pointed in males) and overall body coloration and identified males as type I or type II based on their body size and dissection of supposed type II males to confirm male type (Brantley \& Bass, 1994). We further distinguished between guarding and cuckolder type I males based on their 
Table 1.

An estimate of the proportion of type I males in a population of plainfin midshipman (Porichthys notatus) from British Columbia.

\begin{tabular}{lcccc}
\hline Year & \multicolumn{3}{c}{ Number of males } & $q$ \\
\cline { 2 - 4 } & Type I & Type II & Total & \\
\hline 2008 & 140 & 10 & 150 & 0.93 \\
2009 & 114 & 22 & 136 & 0.84 \\
2010 & 254 & 15 & 269 & 0.94 \\
Total & 508 & 47 & 555 & 0.92 \\
\hline
\end{tabular}

$q$ is the proportion of type I males in our population sample.

position in the nest, because cuckolder males are most often found on the periphery of the nest facing outwards with their urogenital papilla pointing inwards (Lee \& Bass, 2004; K.C. and S.B. personal observation). These characteristics used to identify sex and reproductive type have been verified in extensive studies and field observations have been confirmed through dissections (Bass \& Marchaterre, 1989; Bass \& Anderson, 1991; Brantley \& Bass, 1994).

We collected a small amount of fin tissue from each adult sampled and preserved the tissue in $95 \%$ ethanol. In addition, from a subset of nests ( $N=47$ ), we collected and preserved 40-50 offspring from each cohort found in each nest. A cohort was defined as a distinct group of offspring laid by one or more females in relative synchrony such that the offspring were at the same developmental stage (see Cogliati et al., 2013 for the unique characteristics used to classify developmental stage in midshipman young). Plainfin midshipman nests typically contain between 1 and 4 cohorts. Multiple cohorts were genotyped to capture temporal variation in paternity and cuckoldry. After sampling, we returned the adults (except for type II males) to the nest and then repositioned all rocks to their original position.

\subsection{Genetic analyses}

We genotyped adults and offspring collected across the three years and three beaches using six microsatellite loci developed from plainfin midshipman (loci: Pon22, Pon23, Pon25, Pon30, Pon32 and Pon47; see Suk et al., 2009 for primer sequences). Across the 47 nests from which offspring were collected, we genotyped 1713 offspring (approximately 25 offspring per cohort 
per nest) using the six microsatellite loci (see Cogliati et al., 2013). Additionally, to determine the degree of type I male cuckoldry, here, we focused our genotyping on one well sampled beach in one year (Ladysmith Inlet in 2010; henceforth called LSI2010) and genotyped all adults sampled from this site, for a total of 102 adults (20 females, 78 type I males and 4 type II males). We conducted PCR amplifications following the protocol described in Suk et al. (2009), and conducted fragment analyses on a CEQ 8000 sequencer (Beckman Coulter, Fullerton, CA, USA).

\subsection{Paternity analyses}

We have previously published the paternity results for midshipman males for the 47 genotyped nests, including those from LSI2010, using the twosex paternity model (Neff et al., 2000a, b; Neff, 2001) and COLONY (v2.0; Wang, 2004; Jones \& Wang, 2010; see Cogliati et al., 2013). Across nests, we did not find a difference in paternity between the two models. We used the COLONY assignments in this study because this program generates putative parental genotypes that we could use to identify parent offspring relationships (as opposed to the overall proportional assignments generated by the two-sex paternity model). Unique to this study, we were able to use COLONY to assign cuckolded offspring to individual type I and type II males collected during our intense sampling at the LSI2010 site.

\subsection{Applying the Gross \& Charnov life history model}

We used the Gross \& Charnov (1980) life history model to calculate the relative fitness of type I and type II males. This model uses the proportion of males in the population that develop into type I $(q)$ and the proportion of eggs fertilized in a reproductive season by all type I males $(h)$ to calculate the relative fitness of the two male tactics:

$$
\frac{\omega_{\mathrm{I}}}{\omega_{\mathrm{II}}}=\frac{(1-q) \times h}{q \times(1-h)},
$$

where $\omega$ denotes the fitness of type I males (I) or type II males (II). Thus, the two life histories have equal fitness $\left(\omega_{\mathrm{I}}=\omega_{\mathrm{II}}\right)$ when the proportion of type I males in the population equals the proportion of all eggs fertilized by type I males (i.e., $q=h$; see derivation in Gross \& Charnov, 1980).

Using the data collected from our population monitoring, we calculated $q$ as the proportion of type I males among all males (type I and type II) sampled in the population across the three breeding seasons. During the 
breeding season, type II males are commonly found in nests where females are spawning whereas only juveniles are found in nearby offshore areas (Bass, 1992; Brantley \& Bass, 1994). Thus, it is unlikely that our sampling method strongly biases against type II males. Furthermore, although we sampled reproductive adults that already diverged into each tactic as opposed to the males at the point of divergence, we are assuming minimal differential survivorship between tactics. This seems reasonable in plainfin midshipman because type I males sexually mature only 4 months after type II males (Bass, 1996; Bass et al., 1996).

Because $h$ denotes the proportion of all eggs fertilized by type I males in the population, we needed to incorporate the proportion of eggs sired by type I male cuckolders. To do this, we first defined $h_{\text {guarding }}$ as the proportion of offspring that were assigned by COLONY to nest-guarding males (of a possible 1713 genotyped offspring). We then defined $h_{\text {cuckolder }}$ as the proportion of cuckolded eggs that were also sired by type I males. We estimated this latter value based on the 512 offspring sampled from one location, LSI2010, of which 132 were cuckolded eggs. We calculated $h_{\text {cuckolder }}$ using this subset of offspring because we were able to genotype many adults at LSI2010, and these 512 offspring were included in the initial 1713 used to estimate $h_{\text {guarding. }}$ COLONY was unable to assign all cuckolded offspring to known males, indicating that we did not sample all possible sires (67 of the 132 cuckolded offspring were left unassigned in the LSI2010 samples). Thus, we considered three scenarios. First, we assumed that all 67 offspring of unknown paternity were sired by type II males and $h_{\text {cuckolder }}$ would then equal only the proportion of cuckolder offspring assigned to known, nonnest guarding, type I males in our sample (55 of $\left.132 ; h_{\text {cuckolder_low }}\right)$. Second, we assumed that all 67 offspring of unknown paternity were sired by type I males and $h_{\text {cuckolder }}$ would then equal the proportion of cuckolder offspring assigned to known, non-nest guarding, type I males in addition to all of the offspring assigned to the unknown males $(55+67=122$ of 132 ; $\left.h_{\text {cuckolder_high }}\right)$. Given these two scenarios, $h$ is bounded by:

$$
\begin{aligned}
& h_{\text {low }}=h_{\text {guarding }}+\left(1-h_{\text {guarding }}\right) \times h_{\text {cuckolder_low }} ; \quad \text { and } \\
& h_{\text {high }}=h_{\text {guarding }}+\left(1-h_{\text {guarding }}\right) \times h_{\text {cuckolder_high } .}
\end{aligned}
$$

Third, we assumed that the proportion of cuckoldry performed by type I and type II males in the unassigned offspring was equivalent to the proportion of 
each male's cuckoldry calculated from the offspring that could be assigned (we called this our 'proportional' scenario):

$$
h_{\text {prop }}=h_{\text {guarding }}+\left(1-h_{\text {guarding }}\right) \times h_{\text {cuckolder_prop }} .
$$

\subsection{Statistical analyses}

Using equation (1), we calculated the relative fitness $\left(\omega_{\mathrm{I}} / \omega_{\mathrm{II}}\right)$ of type I males versus type II males for $h_{\text {low }}$ and $h_{\text {high }}$ scenarios, as well as the proportional scenario. To obtain confidence intervals for the three relative fitness estimates, we performed Monte Carlo analyses that re-sampled with replacement the original data at the level of the individual offspring or adult for each variable ( $\left.q, h_{\text {guarding }}, h_{\text {cuckolder_low }}, h_{\text {cuckolder_high }}, h_{\text {cuckolder_prop }}\right)$. Specifically, confidence parameters for $q$ were estimated by re-sampling the adult males, $h_{\text {guarding }}$ were estimated by re-sampling the 1713 offspring, and $h_{\text {cuckolder_low }}$,

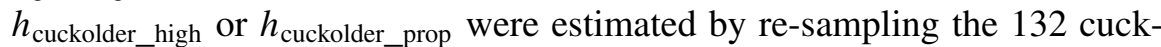
older offspring given the three scenarios for assigning offspring with unknown sires. Each resampled estimate was also used to recalculate the relative fitness $\left(\omega_{\mathrm{I}} / \omega_{\mathrm{II}}\right)$. We performed this procedure 10000 times and used the resulting values to obtain $95 \%$ confidence intervals for the parameters and for the relative fitness estimate. See supplemental material for a summary of values used to calculate fitness variables.

We also conducted a sensitivity analysis to determine where in the bounds fitness changed from type I males having higher fitness to equal fitness to type II males having higher fitness. This analysis was done by resampling the 132 cuckolder offspring in 67 subsequent Monte Carlo analyses, to titrate through the 67 offspring, assigning a different number to type I males in each analysis. We started the Monte Carlo analyses with 0 of the 67 offspring assigned to type I males and ended with 67 of 67 offspring assigned to type I males. For each assignment, we calculated the relative fitness and confidence interval. As an example, assignments for which the subsequent confidence interval included 1.0 defined a bin where the two male types have equal fitness.

\section{Results}

Across the three years, we sampled 555 adult male midshipman, of which 508 were type I males (Table 1). The Monte Carlo simulation revealed that the mean proportion of type I males in the population $(q)$ was 0.92 with a $95 \%$ confidence interval of $0.89-0.94$. In the 47 nests that we ex- 
amined parentage, 1193 of the 1713 genotyped offspring were assigned by COLONY to a guarding type I male. Thus, the proportion of eggs fertilized by guarding type I males, or $h_{\text {guarding, }}$, is equal to 0.70 with a $95 \%$ confidence interval of $0.67-0.72$. This assignment accounts for the occurrence of takeovers and assigns paternity to any previous nest holding type I male (paternity estimate $<10 \%$, see Cogliati et al., 2013). By examining the 512 offspring genotyped from our well sampled LSI2010 site which consisted of 21 cohorts in 8 nests from across the breeding season, we were able to determine the degree of type I male cuckoldry (Table 2). In this sample, 380

Table 2.

Summary of paternity assignments for plainfin midshipman (Porichthys notatus) collected at one beach in Ladysmith Inlet during 2010 using COLONY.

\begin{tabular}{|c|c|c|c|c|c|c|}
\hline \multirow[t]{3}{*}{ Nest } & \multirow[t]{3}{*}{ Cohort } & \multirow[t]{3}{*}{ Offspring } & \multicolumn{4}{|c|}{ Paternity } \\
\hline & & & \multirow[t]{2}{*}{ Guarding } & \multicolumn{3}{|c|}{ Cuckoldry } \\
\hline & & & & Type I & Type II & Unknown \\
\hline \multirow[t]{2}{*}{1} & 1 & 25 & 21 & 0 & 3 & 1 \\
\hline & 2 & 23 & 23 & 0 & 0 & 0 \\
\hline \multirow[t]{3}{*}{2} & 1 & 24 & 6 & 3 & 0 & 15 \\
\hline & 2 & 26 & 21 & 1 & 2 & 2 \\
\hline & 3 & 26 & 0 & $12^{*}$ & 0 & 14 \\
\hline 3 & 1 & 25 & 10 & 0 & 0 & 15 \\
\hline \multirow[t]{2}{*}{4} & 1 & 23 & 23 & 0 & 0 & 0 \\
\hline & 2 & 24 & 9 & 9 & 0 & 6 \\
\hline \multirow[t]{4}{*}{5} & 1 & 24 & 19 & 1 & 3 & 1 \\
\hline & 2 & 24 & 20 & 4 & 0 & 0 \\
\hline & 3 & 25 & 23 & 0 & 0 & 2 \\
\hline & 4 & 25 & 23 & 0 & 0 & 2 \\
\hline \multirow[t]{3}{*}{6} & 1 & 25 & 23 & 0 & 2 & 0 \\
\hline & 2 & 22 & 22 & 0 & 0 & 0 \\
\hline & 3 & 25 & 24 & 1 & 0 & 0 \\
\hline \multirow[t]{3}{*}{7} & 1 & 24 & 21 & 0 & 0 & 3 \\
\hline & 2 & 25 & 25 & 0 & 0 & 0 \\
\hline & 3 & 24 & 0 & $24^{*}$ & 0 & 0 \\
\hline \multirow[t]{3}{*}{8} & 1 & 24 & 24 & 0 & 0 & 0 \\
\hline & 2 & 24 & 18 & 0 & 0 & 6 \\
\hline & 3 & 25 & 25 & 0 & 0 & 0 \\
\hline Total & & 512 & 380 & 55 & 10 & 67 \\
\hline
\end{tabular}

Paternity represents the numbers of offspring assigned to each male type.

* Multiple known type I males are responsible for siring these offspring. 
offspring were assigned to a guarding type I male, leaving 132 cuckolded offspring. Of the 132 cuckolder offspring, COLONY assigned 55 to known type I males, 10 to known type II males, and 67 to unknown males (i.e., males not sampled; Table 2). Altogether, we were able to assign 445 of the 512 offspring (87\%) to males sampled in our population from our well sampled LSI2010 site.

If we assume that all 67 offspring of unknown paternity were sired by type II males, we generate an $h_{\text {cuckolder_low }}$ that equals $0.42(95 \% \mathrm{CI}=$ $0.33-0.50)$. The proportion of offspring fertilized by all type I males $\left(h_{\text {low }}\right)$ would then equal $0.82(95 \% \mathrm{CI}=0.79-0.85)$, and the relative fitness of the two male tactics $\omega_{\text {I }} / \omega_{\text {II_low }}$ would be 0.44 , with a $95 \%$ confidence interval of 0.29-0.61 (Figure 1). If instead, all 67 offspring of unknown paternity were sired by type I males, we generate an $h_{\text {cuckolder_high }}$ that equals 0.92 (95\% CI $=0.88-0.97$ ) and the proportion of offspring fertilized by type I males $\left(h_{\text {high }}\right)$ would equal $0.98(95 \% \mathrm{CI}=0.96-0.99)$. The relative fitness $\omega_{\mathrm{I}} / \omega_{\text {II_high }}$ would be 4.5 with a $95 \%$ confidence interval of $2.1-9.4$ (Figure 1). Finally, if we assume the proportional scenario, where $85 \%$ or 57 of the 67 offspring of unknown paternity were sired by type I males (based on 55 type I offspring out of 65 known cuckolder offspring), then $h_{\text {cuckolder_prop }}$ equals 0.85 (95\% CI $=0.79-0.91)$. The proportion of offspring fertilized by type I males $\left(h_{\text {prop }}\right)$ would then equal $0.95(95 \% \mathrm{CI}=0.93-0.97)$, and the relative fitness $\omega_{\text {I }} / \omega_{\text {II_prop }}$ would be 2.0 with a $95 \%$ confidence interval of 1.2-3.4 (Figure 1). Thus, if the unknown paternity is attributed entirely to type I males, or if $85 \%$ is attributed to them (our proportional scenario), then the fitness of type I males is significantly higher than that of type II males. Conversely, of our three scenarios, it is only when all of the unknown paternity is attributed entirely to type II males that the fitness of type I males is significantly lower than that of type II males.

We then used our sensitivity analysis to titrate through the number of offspring that were assigned to unknown males, to determine the critical points where relative fitness between the two tactics would be equal. We found that when 27 or fewer of the possible 67 unassigned offspring were sired by type I males, the relative fitness of type I males was significantly less than 1 . At the other extreme, when 52 or more of the possible 67 offspring were sired by type I males, the relative fitness of type I males was significantly greater than 1. No significant difference in the relative fitness of type I and type II males was observed when 28 to 51 of the offspring sired by unknown males were assigned to type I males. 


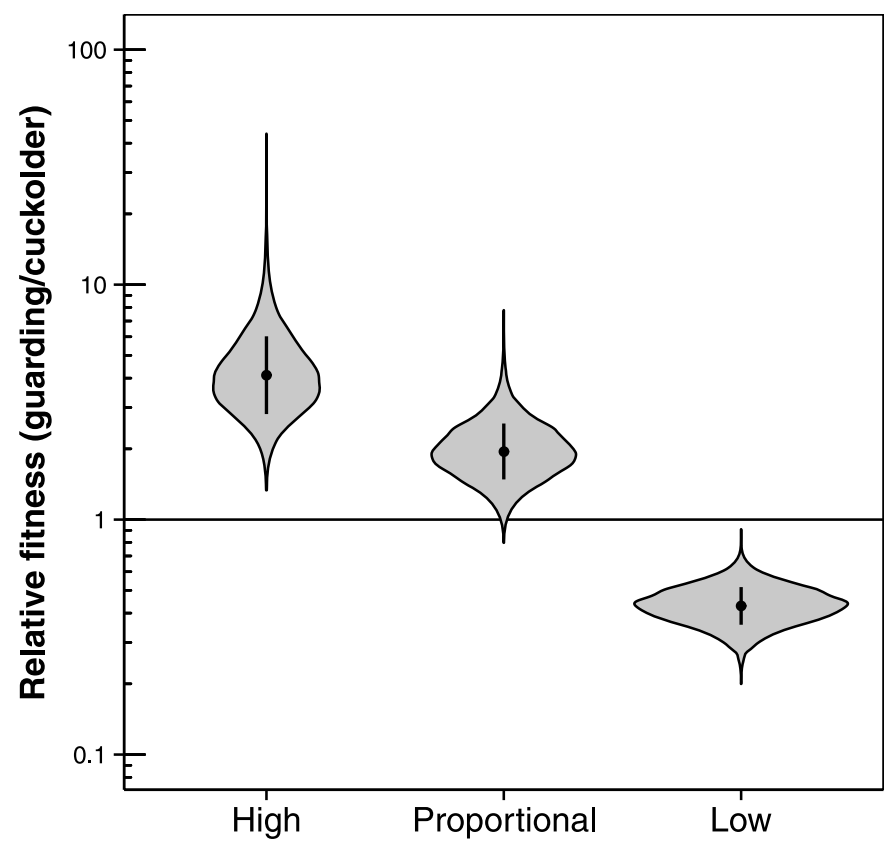

Figure 1. Estimates of the relative fitness of alternative reproductive tactics in plainfin midshipman (Porichthys notatus). Shown are the mean relative fitness $( \pm 1 \mathrm{SD}$, dot and vertical line) for type I males given high (all unknown sires as type I), proportional (85\% of unknown sires as type I), and low (all unknown sires as type II) scenarios (see text for details). The violin plots show the frequency histograms of the distribution of data from the Monte Carlo analyses. The width of the violin plots are an outcome of re-sampling the data and applying equation (1). Data are bounded between zero and one when type I males have lower fitness, while there is no upper bound in the equation when type I males have higher fitness. The solid horizontal line depicts equal fitness $(=1)$ between type I and type II males. The $y$-axis is a $\log$ scale to better depict the ratio data.

\section{Discussion}

In this study, we conducted intense field sampling and used genetic markers to explore patterns of paternity to estimate the degree of cuckoldry in the plainfin midshipman, a species with well-described alternative reproductive tactics. Our analyses built on those presented in Cogliati et al. (2013), who first estimated cuckoldry rates in this system and documented nest takeover behaviour by type I males. Here, we used extensive sampling at the Ladysmith Inlet site (LSI2010) to partition the observed paternity loss between specialized cuckolder males (type II) and opportunistic cuckoldry performed by type I males. Surprisingly, based on our samples, we found that nearly 
$42 \%$ of all documented cuckoldry was done by type I males (fathering $11 \%$ of all offspring at this site), whereas only $7 \%$ was done by known type II males ( $2 \%$ of all offspring at this site). The remaining $51 \%$ of the cuckolded offspring could not be assigned to any of the males that we sampled (13\% of all offspring at this site). Our results thereby corroborate observations of cuckoldry behaviour by type I males reported in Lee and Bass (2004), and provide the first estimate of the frequency of cuckoldry by type I males in plainfin midshipman. Examples of such behavioural flexibility within a male tactic are restricted to species where male alternative tactics are distinct. For example, a type I male may display cuckoldry behaviour, but he is still morphologically considered a type I male and not a type II male. Such within tactic variation cannot be determined in species where males display flexible tactics and do not have distinct morphs, because each male is morphologically similar and is capable of displaying either tactic (e.g., a male guppy can mate through courtship or forced copulation; Magurran \& Seghers, 1991, 1994). We know of only one other study that has reported estimates of paternity loss from competition within a tactic type, not including takeovers or abandonment. In bluegill, Neff (2001) documented paternity loss of $1.8 \%$ to other parental males who are cuckolding. This high occurrence of flexible cuckoldry in plainfin midshipman may reflect greater nest site limitations and the protracted parental care period (3-4 months), which may considerably increase the benefit of type I cuckoldry compared to the situation for bluegill (Cogliati et al., 2013). Thus, our genetic analyses have documented several reproductive behaviours used by type I males, comprising guarding, nest takeovers, and cuckoldry.

We were also able to use our genetic data to estimate the relative fitness of the alternative reproductive tactics in plainfin midshipman. The life history model developed by Gross \& Charnov (1980) provides a useful method for calculating the relative fitness of alternative reproductive tactics. Male alternative reproductive tactics can arise from a genetic polymorphism or from a conditional strategy, with the former mechanism predicting equal fitness between the tactics and the latter predicting that one tactic will have higher fitness (see Gross, 1996). Applying the life history model to our multiyear data indicated that the relative fitness of type I males had an upper estimate of 4.5 and a lower estimate of 0.44 , where a relative fitness of 1 indicates equal fitness for the two male tactics. Our sensitivity analyses revealed that type I males had lower fitness than type II males when fewer 
than $62 \%$ of all cuckolded offspring were sired by type I males, equal fitness when $63 \%$ to $80 \%$ of cuckolded offspring were sired by type I males, and greater fitness when more than $81 \%$ of the offspring were sired by type I males. At first pass, our analysis is equivocal, encompassing higher, equal, and lower fitness for type I males.

However based on two lines of evidence, we argue that type I males are most likely to have higher or equal fitness compared to type II males. First, type I males sired more than 2 times as many of the cuckolded offspring that could be assigned to known males than did type II males. Of the offspring that were assigned to cuckolders, type I males sired an average of 5.5 offspring per cohort (range 1-24), compared to type II males who sired an average of 2.5 offspring per cohort (range 2-3). Given this distribution of paternity assignment, with type II males fathering a very small number of offspring per cohort, we can reasonably assume that any male that sired 6 or more offspring in a cohort was likely to be a type I male. Of the unassigned offspring, the reconstructed genotypes provided by COLONY found 22 distinct sires of which four had sired 6 or more offspring within a cohort (overall range 1-12; data not shown) for a total of 35 offspring. If we consider these four unknown males who sired a relatively large number of young to be type I males, then type I males would account for at least 35 of the 67 unknown offspring and type I males would have equal or higher fitness compared to type II males. Second, it is likely that type I males sired more than $77 \%$ (52 of 67) of the unassigned offspring. For example, based on the 65 assigned cuckolded offspring, type I males sired 85\% (=55/65). Assuming the same proportion (0.85) in the unassigned offspring would give 57 of the 67 unassigned offspring to type I males (our proportional scenario), and supports the notion that type I males have higher fitness than type II males. Furthermore, the Gross \& Charnov (1980) model assumes that $q$ is based on the number of males at the point of tactic divergence, and while we were unable to sample males at this point, $q$ would have to equal 0.98 or greater to shift our prediction such that type II males have higher fitness. Thus, although we cannot conclusively differentiate between alternative strategies and a conditional strategy, our data do suggest that type I males have equal or higher fitness and it is type II males that might be making the best of a bad situation (sensu Dawkins, 1980; Eberhard, 1982).

Understanding the evolution of alternative reproductive tactics has been an area of great interest for decades, yet testing the prediction of equal fitness has only been done in a handful of systems (Gross \& Charnov, 1980; Shus- 
ter \& Wade, 1991; Ryan et al., 1992; Sinervo \& Lively, 1996; Neff \& Lister, 2007; Rios-Cardenas \& Webster, 2008). In comparison to the hundreds of species with documented alternative reproductive tactics, the relative paucity of tests for the prediction of equal or unequal fitness is likely due, in part, to the challenges of measuring reproductive success for each male tactic, particularly when reproductive success fluctuates in both space and time (e.g., Neff \& Clare, 2008; Cogliati et al., 2013). In the extensively studied bluegill, Neff \& Lister (2007) observed that the cuckolder morph had slightly higher fitness than the parental morph, suggesting a conditional strategy. Their result differed from the initial conclusions by Gross \& Charnov (1980), who calculated equal fitness between the two morphs in the same population. This discrepancy was largely driven by the inclusion of differential survivorship of offspring sired by parental versus cuckolder males in the Neff \& Lister (2007) study, where cuckolder offspring had significantly higher survivorship than parental offspring prior to differentiation into the two life histories. The Gross \& Charnov (1980) model assumes equal survivorship of offspring prior to differentiation into the two tactics. Indeed, in our study we were unable to include survivorship data in the relative fitness calculation. However, because the tactics in plainfin midshipman differentiate at approximately 200 days of age and type I males are known to mature just a few months after type II males (Bass et al., 1996), we doubt there will be a major effect of differential survivorship of the offspring on the calculation of fitness of the two tactics. Bluegill tactics, in comparison, diverge at 2 years of age when sneakers first mature (Gross, 1982). Therefore, the impacts of differential survivorship are likely considerably less severe in plainfin midshipman. Nevertheless, our assumption of similar survivorship to tactic divergence remains to be empirically tested in plainfin midshipman.

In this study, we investigated whether the plainfin midshipman male reproductive tactics evolved via one of two mutually exclusive possibilities: genetic or conditional strategies (Gross, 1996). In a recent review, Neff \& Svensson (2013) proposed a model termed 'conditional alternative strategies', which treats the two approaches (genetic and conditional) as two ends of a single continuum and thus combines both genetic and environmental effects on tactic expression. Given that approach, it is possible to observe additive genetic variance in condition dependent 'thresholds' (Piche et al., 2008; Tomkins \& Moczek, 2009) or 'switch points' (Roff, 2011). The plainfin midshipman would be an ideal species to test the model developed by 
Neff \& Svensson (2013). For example, those authors suggest the use of natural breeding experiments and multiple rearing environments to investigate gene-by-environment interactions (i.e., interactions between sire life history, or genes, and the environment, such as food availability, predation risk, or competition). These experiments can be executed with relative ease in plainfin midshipman, because they can be sampled readily at low tide and have an extended breeding season. Furthermore, sexual maturity and tactic differentiation occurs at a relatively young age for midshipman (Bass et al., 1996) and therefore monitoring or manipulating aspects of the environment before tactic differentiation occurs could be performed and then evaluated. This approach could get at a gene-by-environment interaction, which is a key predictor of the Neff \& Svensson (2013) model.

In conclusion, the results of our study delineate the possible upper and lower bounds of relative fitness between the two male tactics seen in plainfin midshipman, encompassing both traditional mechanisms for the evolution of alternative reproductive tactics: genetically determined alternative strategies or a single conditional strategy. Based on how rare and unsuccessful type II males were at our extensively sampled site, we argue that type I males are likely to have higher fitness to type II males. In addition, we show that type I males cuckold at a relatively high frequency, adding to the repertoire of reproductive behaviours used by these males. Given the tractability of the midshipman system, a valuable next step will be to look for gene-byenvironment interactions on tactic development and expression as predicted by the conditional alternative strategies model of Neff \& Svensson (2013).

\section{Acknowledgements}

We thank Shawn Garner and John Fitzpatrick for valuable comments on the manuscript. We thank Celia Chui, John Fitzpatrick, Ahdia Hassan, Julie Marentette, Jennifer Reynolds, Natalie Sopinka and Jonathan Taves for their assistance with data collection in British Columbia. Michael Roubakha, Shawn Garner, Cayli Hunt, Celeste Bouchard and Amanda Pereira assisted with DNA extractions and sample analyses. Ben Bolker provided advice on the data analysis and Shawn Garner wrote the Excel macros to conduct the Monte Carlo analyses. We also thank Mr. and Mrs. Cogswell, Mr. and Mrs. Weatherell, and especially Mr. and Mrs. Flader for providing access to midshipman habitats and lodgings for field crew. This work was funded by the 
Natural Sciences and Engineering Research Council of Canada (grants to S.B. and B.D.N.).

\section{References}

Arora, H.L. (1948). Observations on the habits and early life history of the Batrachoid fish, Porichthys notatus Girard. - Copeia: 89-93.

Bass, A.H. (1990). Sounds from the intertidal zone: vocalizing fish. — BioScience 40: 247258.

Bass, A.H. (1992). Dimorphic male brains and alternative reproductive tactics in a vocalizing fish. - Trends Neurosci. 15: 139-145.

Bass, A.H. (1993). From brains to behaviour: hormonal cascades and alternative mating tactics in teleost fishes. - Rev. Fish Biol. Fisheries 3: 181-186.

Bass, A.H. (1996). Shaping brain sexuality. - Am. Sci. 84: 352-363.

Bass, A.H. \& Anderson, K. (1991). Intra- and inter-sexual dimorphisms in the sound generating motor system in a vocalizing fish: motor axon number and size. — Brain Behav. Evol. 37: 204-214.

Bass, A.H., Horvath, B.J. \& Brothers, E.B. (1996). Nonsequential developmental trajectories lead to dimorphic vocal circuitry for males with alternative reproductive tactics. J. Neurobiol. 30: 493-504.

Bass, A.H. \& Marchaterre, M.A. (1989). Sound-generating (sonic) motor system in a teleost fish (Porichthys notatus): sexual polymorphisms and general synaptology of sonic motor nucleus. — J. Comp. Neurol. 86: 154-169.

Brantley, R.K. \& Bass, A.H. (1994). Alternative male spawning tactics and acoustic signals in the plainfin midshipman fish Porichthy notatus Girard (Teleostei, Batrachoididae). Ethology 96: 213-232.

Brantley, R.K., Marchaterre, M.A. \& Bass, A.H. (1993). Androgen effects on vocal muscle structure in a teleost fish with inter- and intra-sexual dimorphism. - J. Morph. 216: 305318.

Cogliati, K.M., Neff, B.D. \& Balshine, S. (2013). High degree of paternity loss in a species with alternative reproductive tactics. — Behav. Ecol. Sociobiol. 67: 399-408.

Dawkins, R. (1980). Good strategy or evolutionarily stable strategy? — In: Sociobiology: beyond nature/nurture? (Silverberg, J., ed.). Westview Press, CO, p. 331-367.

DeMartini, E.E. (1988). Spawning success of the male plainfin midshipman. I. Influences of male body size and area of spawning site. - J. Exp. Mar. Biol. Ecol. 121: 177-192.

DeMartini, E.E. (1990). Annual variations in fecundity, egg size and condition of the plainfin midshipman (Porichthys notatus). - Copeia: 850-855.

Eberhard, W.G. (1994). Beetle horn dimorphism: making the best of a bad lot. — Am. Nat. 119: 420-426.

Emlen, D.J. (1994). Environmental control of horn length dimorphism in the beetle Onthophagus acuminatus (Coleoptera: Scarabaeidae). — Proc. Roy. Soc. Lond. B: Biol. Sci. 256: 131-136. 
Emlen, D.J. (2008). The roles of genes and the environment in the expression and evolution of alternative tactics. - In: Alternative reproductive tactics (Oliveira, R.F., Taborsky, M. \& Brockman, H.J., eds). Cambridge University Press, New York, NY, p. 85-108.

Foran, C.M. (1998). Phenotypic plasticity in the neuroendocrine axis of a teleost fish with alternative reproductive tactics (Porichthys notatus). - PhD Dissertation, Cornell University, Ithaca, NY.

Gross, M.R. (1982). Sneakers, satellites and parentals: polymorphic mating strategies in North American sunfishes. - Z. Tierpsychol. 60: 1-26.

Gross, M.R. (1996). Alternative reproductive strategies and tactics: diversity within sexes. Trends Ecol. Evol. 11: 92-98.

Gross, M.R. \& Charnov, E.L. (1980). Alternative male life histories in bluegill sunfish. Proc. Natl. Acad. Sci. USA 77: 6937-6940.

Hubbs, C.L. (1920). The bionomics of Porichthy notatus Girard. - Am. Nat. 54: 380-384.

Ibara, R.M., Penny, L.T., Ebeling, A.W., van Dykhuizen, G. \& Caillet, G. (1983). The mating call of the plainfin midshipman fish, Porichthys notatus. — In: Predators and prey in fishes (Ward, J.A., ed.). Dr. W. Junk Publishers, The Hague, p. 205-212.

Jones, O.R. \& Wang, J. (2010). Colony: a program for parentage and sibship inference from multilocus genotype data. - Mol. Ecol. Resources 10: 551-555.

Lank, D.B., Smith, C.M., Hanotte, O., Burke, T. \& Cooke, F. (1995). Genetic polymorphism for alternative mating behavior in lekking male ruff Philomachus pugnax. - Nature 378: 59-62.

Lee, J.S.F. \& Bass, A.H. (2004). Does exaggerated morphology preclude plasticity to cuckoldry in the midshipman fish (Porichthys notatus). — Naturwissenschaften 91: 338-341.

Magurran, A.E. \& Seghers, B.H. (1991). Variation in schooling and aggression behaviour amongst guppy (Poecilia reticulata) populations in Trinidad. — Behaviour 118: 215-234.

Magurran, A.E. \& Seghers, B.H. (1994). Sexual conflict as a consequence of ecology: evidence from guppy, Poecilia reticulata, populations in Trinidad. — Proc. Roy. Soc. Lond. B: Biol. Sci. 255: 31-36.

Maynard Smith, J. (1982). Evolution and the theory of games. - Cambridge University Press, Cambridge.

Miller, D.J. \& Lea, R.N. (1972). In: Fish Bulletin 157: guide to the coastal marine fishes of California. - Scripps Institution of Oceanography, UC San Diego, San Diego, CA, p. $72-73$.

Neff, B.D. (2001). Genetic paternity analysis and breeding success in bluegill sunfish (Lepomis macrochirus). — J. Hered. 92: 111-119.

Neff, B.D. \& Clare, E.L. (2008). Temporal variation in cuckoldry and paternity in two sunfish species (Lepomis spp.) with alternative reproductive tactics. — Can. J. Zool. 86: 92-99.

Neff, B.D. \& Lister, J.S. (2007). Genetic life history effects on juvenile survival in bluegill. — J. Evol. Biol. 20: 517-525.

Neff, B.D. \& Svensson, E.I. (2013). Polyandry and alternative mating tactics. - Phil. Trans. Roy. Soc. B 368: 20120045.

Neff, B.D., Repka, J. \& Gross, M.R. (2000a). Parentage analysis with incomplete sampling of candidate parents and offspring. - Mol. Ecol. 9: 515-528. 
Neff, B.D., Repka, J. \& Gross, M.R. (2000b). Statistical confidence in parentage analyses with incomplete sampling: how many loci and offspring are needed? - Mol. Ecol. 9: 529-539.

Oliveira, R.F., Taborsky, M. \& Brockmann, H.J. (eds) (2008). Alternative reproductive tactics: an integrative approach. - Cambridge University Press, New York, NY.

Piche, J., Hutchings, J.A. \& Blanchard, W. (2008). Genetic variation in threshold reaction norms for alternative reproductive tactics in male Atlantic salmon, Salmo salar. - Proc. Roy. Soc. Lond. B: Biol. Sci. 275: 1571-1575.

Rios-Cardenas, O. \& Webster, M.S. (2008). A molecular genetic examination of the mating system of pumpkinseed sunfish reveals high pay-offs for specialized sneakers. - Mol. Ecol. 17: 2310-2320.

Roff, D.A. (2011). Alternative strategies: the evolution of switch points. - Curr. Biol. 21: R285-R287.

Ryan, M.J., Pease, C.M. \& Morris, M.R. (1992). A genetic polymorphism in the swordtail Xiphophorus nigrensis: testing the prediction of equal fitnesses. - Am. Nat. 139: 21-31.

Shuster, S.M. (1989). Male alternative reproductive strategies in a marine isopod crustacean (Paracerceis sculpta): the use of genetic markers to measure differences in fertilization success among $\alpha$-, $\beta$-, and $\gamma$-males. — Evolution 43: 1683-1698.

Shuster, S.M. \& Wade, M.J. (1991). Equal mating success among male reproductive strategies in a marine isopod. - Nature 350: 608-610.

Sinervo, B. \& Lively, C.M. (1996). The rock-paper-scissors game and the evolution of alternative male strategies. - Nature 380: 240-243.

Suk, H.Y., Neff, B.D., Fitzpatrick, J.L. \& Balshine, S. (2009). Isolation and characterization of polymorphic microsatellite loci in the plain midshipman fish. - Hereditas 146: 001004.

Taborsky, M., Oliveira, R.F. \& Brockman, H.J. (2008). The evolution of alternative reproductive tactics: concepts and questions. - In: Alternative reproductive tactics (Oliveira, R.F., Taborsky, M. \& Brockman, H.J., eds). Cambridge University Press, New York, NY, p. 1-21.

Thornhill, R. (1981). Panorpa (Mecoptera: Panorpidae) scorpionflies: systems for understanding resource-defense polygyny and alternative male reproductive efforts. - Annu. Rev. Ecol. Syst. 12: 355-386.

Tomkins, J.L. \& Moczek, A.P. (2009). Patterns of threshold evolution in polyphonic insects under different developmental models. — Evolution 63: 459-468.

Wang, J. (2004). Sibship reconstruction from genetic data with typing errors. - Genetics 166: 1963-1979. 